\title{
Possible Effect of Concomitant Prokinetics and Herbal Medicines against Nausea in Patients Taking Lubiprostone
}

\author{
Takatsugu Yamamoto, Shun Osumi, Daisuke Yanagisawa, Hiroshi Yamato, \\ Hitoshi Aoyagi, Akari Isono, Koichiro Abe, and Hiroto Kita \\ Department of Internal Medicine, Teikyo University School of Medicine, Tokyo, Japan \\ Correspondence should be addressed to Takatsugu Yamamoto; ymmt@med.teikyo-u.ac.jp
}

Received 27 August 2017; Accepted 26 September 2017; Published 7 December 2017

Academic Editor: Toshimi Chiba

Copyright (C) 2017 Takatsugu Yamamoto et al. This is an open access article distributed under the Creative Commons Attribution License, which permits unrestricted use, distribution, and reproduction in any medium, provided the original work is properly cited.

Background and Aim. Lubiprostone is a novel laxative that sometimes causes nausea, but preventive strategies remain unconfirmed. Methods. We retrospectively chose 126 patients prescribed lubiprostone from 2013 to 2016. Medical records were reviewed to clarify whether nausea developed after administration of the drug. Background characteristics, including concomitant medicines, were also reviewed. Results. The most common adverse symptom was diarrhea (23.8\%). Nausea occurred in 16 patients (12.7\%). Patients taking either prokinetics or herbal medicines or both were unlikely to develop nausea $(p=0.007)$. Conclusions. Concomitant prokinetics and/or herbal medicines may help alleviate lubiprostone-induced nausea.

\section{Introduction}

Chronic constipation (CC) is a common condition that sometimes impairs a patient's quality of life. The number of patients with CC has recently increased in association with the advanced-aged society in developed countries [1]. Lubiprostone is a selective type 2 chloride-channel activator that increases the liquid component in the small intestine, enhances bowel motility, and improves constipation. This agent has become essential for clinicians in the management of patients experiencing CC and constipation-associated irritable bowel syndrome [2-4]. On the other hand, lubiprostone has been reported to sometimes cause upper gastrointestinal symptoms such as nausea and vomiting, which is one of chief reasons for discontinuation of the drug [5]. A possible mechanism is that an increase in luminal volume may cause distension of the small intestine, inducing a delay in gastric emptying and thus a sensation of nausea via vagal stimulation $[6,7]$. However, it is difficult to predict the development of such troublesome symptoms before administration, and strategies for prevention remain uncertain. We conducted the present study to identify the key clinical factors associated with the development of lubiprostone-induced nausea.

\section{Materials and Methods}

This is a retrospective study using the medical records of patients in Teikyo University Hospital (Tokyo, Japan). First, all 269 patients who had been prescribed lubiprostone (Amitiza $^{\circledR}$, Mylan EPD, Tokyo) for CC in the Department of Internal Medicine (January 2013 to April 2016) were identified from patient lists. A diagnosis of constipation was made according to the Rome III criteria: bowel movements less than three times per week, hard stools, a sensation of incomplete emptying, or difficulty in evacuation on at least $25 \%$ of occasions [8]. Then, 80 patients with an insufficient description of clinical effectiveness and 63 patients without follow-up after administration were excluded, with 126 patients finally enrolled as subjects. Medical records of the subjects were examined by two authors (T. Y. and H. A.), to judge their responsiveness to lubiprostone and development of adverse effects such as nausea, vomiting, diarrhea, or others within 1 month (as the endpoints of the study). If the drug was discontinued, the cause was identified, where possible. In addition, clinical data such as demographics and concomitant medications were examined to evaluate their influence on responsiveness and the development of adverse reactions. 
TABLE 1: Background characteristics and clinical response to lubiprostone.

\begin{tabular}{lc}
\hline Number of patients & 126 \\
Age (year \pm SD) & $65.4 \pm 15.7$ \\
Sex (male/female) & $61 / 65$ \\
Mental disorder & $13(10.3 \%)$ \\
Concomitant agents & $69(54.8 \%)$ \\
$\quad$ Laxatives & $56(44.4 \%)$ \\
$\quad$ Prokinetics/herbal medicines & $10(7.9 \%)$ \\
$\quad$ Probiotics & $33(26.2 \%)$ \\
$\quad$ PPI/H2RA & $95(75.4 \%)$ \\
Effectiveness & $30(23.8 \%)$ \\
Diarrhea & $16(12.7 \%)$ \\
Nausea/vomiting & $53(42 \%)$ \\
Discontinuation & \\
\hline
\end{tabular}

Other adverse effects included edema (2 patients), headache (1 patient), and palpitation (1 patient). SD: standard deviation, PPI: proton pump inhibitor, and H2RA: histamine 2 receptor antagonist.

TABLE 2: Relationship between nausea/vomiting after administration of lubiprostone and clinical factors based on univariate analysis.

\begin{tabular}{lccc}
\hline Clinical factor & Odds ratio & 95\% confidence interval & $p$ value \\
\hline Age & 0.956 & $0.924-0.988$ & 0.007 \\
Sex & 0.310 & $0.094-1.021$ & 0.054 \\
Mental disorder & 0.759 & $0.258-6.414$ & 0.759 \\
Laxatives & 0.898 & $0.372-3.082$ & 0.898 \\
Prokinetics/herbal medicines & 0.037 & $0.067-0.920$ & 0.037 \\
Probiotics & 0.790 & $0.088-6.333$ & 0.790 \\
PPI/H2RA & 0.364 & $0.078-1.696$ & 0.198 \\
\hline
\end{tabular}

PPI: proton pump inhibitor and H2RA: histamine 2 receptor antagonist.

In the statistical analysis, a $p$ value $<0.05$ was regarded as significant. To clarify significant clinical factors related to responsiveness, univariate and multivariate analyses were performed using logistic regression analysis. All statistical evaluations were made using SPSS Statistics, version 19 (IBM Japan, Tokyo, Japan). This protocol was approved by the Institutional Review Board of Teikyo University prior to the study (TU-16-018).

\section{Results}

Background characteristics of the study subjects are shown in Table 1. The subjects included 61 men and 65 women, and the average age was 65.4 years. It was noted that female patients were significantly younger than male patients. The rate of concomitant laxatives was almost $50 \%$. The response rate reached up to $75.4 \%$, while adverse effects were seen in about $40 \%$ of all subjects. Common adverse reactions were diarrhea (30 patients, 23.8\%) and nausea, vomiting, or both (16 patients, $12.7 \%)$. Others included edema ( 2 patients), headache ( 1 patient), and palpitations ( 1 patient). The rate of discontinuation reached $42 \%$ of all the subjects. The major reason for discontinuation was an adverse effect such as nausea or diarrhea.

Table 2 shows the impact of clinical factors on responsiveness and adverse effects, using univariate analysis. Effectiveness was significantly related to older age. Regarding adverse effects, no factors were associated with the development of diarrhea. On the other hand, development of nausea was less frequent in elderly patients and in those treated with prokinetics, such as mosapride, itopride, and/or Japanese herbal medicines, including rikkunshito, daikenchuto, mashiningan, or daiou (Rhei rhizoma), than in those without (Table 2). These two groups of medicines were analyzed together because most of them were concomitantly prescribed. Women tended to be more susceptible to nausea than men during lubiprostone treatment. Multivariate analysis showed that age and concomitant prokinetics or herbal medicines were significant factors related to upper gastrointestinal symptoms.

\section{Discussion}

Gastrointestinal symptoms are cumbersome reactions during treatment with lubiprostone. According to previous data, incidence reached $40 \%$, and the most frequent symptom was nausea [5]. In the present study, we found the incidence was similar, though diarrhea was the most common symptom. Unfortunately, because dose reduction has only a partial prophylactic effect against lubiprostone-induced nausea, discontinuation of the agent is necessary, and preventive treatment has not yet been established [9]. In a recent study from Japan, it was reported that itopride, a prokinetic drug with antidopamine effect, might be useful in alleviating 
nausea [10]. In the present study, we showed the possible effectiveness of concomitant herbal medicines. Daikenchuto is an herbal medicine that is effective for bloating in patients with CC, probably via action on the gut microbiota $[11,12]$. Rikkunshito is a ghrelin enhancer and is effective against upper gastrointestinal symptoms $[13,14]$. Daiou, or Rhei rhizoma, has been proven to have an inhibitory effect on reflux esophagitis in rats [15]. Although it remains unclear which specific type of medicines was most effective for preventing nausea because of the small number of subjects in the present study, prokinetics and herbal medicines might help prevent nausea induced by lubiprostone. Further prospective studies are required to clarify clinical efficacy.

Limitations must be considered in the interpretation of the present data. One is the retrospective study design. Second, this study was carried out at a single institution, and third, the sample size was small. Further multicenter prospective studies are required to determine which agents are appropriate for preventing lubiprostone-induced nausea.

\section{Conflicts of Interest}

No authors have conflicts of interest with regard to the present research.

\section{References}

[1] M. V. Roque and E. P. Bouras, "Epidemiology and management of chronic constipation in elderly patients," Clinical Interventions in Aging, vol. 10, pp. 919-930, 2015.

[2] D. A. Drossman, W. D. Chey, J. F. Johanson et al., "Clinical trial: Lubiprostone in patients with constipation-associated irritable bowel syndrome - Results of two randomized, placebocontrolled studies," Alimentary Pharmacology \& Therapeutics, vol. 29, no. 3, pp. 329-341, 2009.

[3] J. F. Johanson, D. A. Drossman, R. Panas, A. Wahle, and R. Ueno, "Clinical trial: Phase 2 study of lubiprostone for irritable bowel syndrome with constipation," Alimentary Pharmacology \& Therapeutics, vol. 27, no. 8, pp. 685-696, 2008.

[4] J. F. Johanson, D. Morton, J. Geenen, and R. Ueno, "Multicenter, 4-week, double-blind, randomized, placebo-controlled trial of lubiprostone, a locally-acting type-2 chloride channel activator, in patients with chronic constipation," American Journal of Gastroenterology, vol. 103, no. 1, pp. 170-177, 2008.

[5] C. F. Barish, D. Drossman, J. F. Johanson, and R. Ueno, "Efficacy and safety of lubiprostone in patients with chronic constipation," Digestive Diseases and Sciences, vol. 55, no. 4, pp. 10901097, 2010.

[6] M. Camilleri, A. E. Bharucha, R. Ueno et al., "Effect of a selective chloride channel activator, lubiprostone, on gastrointestinal transit, gastric sensory, and motor functions in healthy volunteers," American Journal of Physiology-Gastrointestinal and Liver Physiology, vol. 290, no. 5, pp. G942-G947, 2006.

[7] M. Matsuura, M. Inamori, H. Endo et al., "Lubiprostone decreases the small bowel transit time by capsule endoscopy: an exploratory, randomised, double-blind, placebo-controlled 3way crossover study," Gastroenterology Research and Practice, vol. 2014, Article ID 879595, 6 pages, 2014.

[8] G. F. Longstreth, W. G. Thompson, W. D. Chey, L. A. Houghton, F. Mearin, and R. C. Spiller, "Functional bowel disorders," Gastroenterology, vol. 130, no. 5, pp. 1480-1491, 2006.
[9] B. Gras-Miralles and F. Cremonini, "A critical appraisal of lubiprostone in the treatment of chronic constipation in the elderly," Clinical Interventions in Aging, vol. 8, pp. 191-200, 2013.

[10] T. Abe, Y. Hachiro, Y. Ebisawa, H. Hishiyama, M. Murakami, and M. Kunimoto, "Efficacy of itopride in the prevention of lubiprostone-induced nausea," Open Journal of Gastroenterology, vol. 04, no. 09, pp. 305-309, 2014.

[11] M. Yuki, Y. Komazawa, Y. Kobayashi et al., "Effects of Daikenchuto on Abdominal Bloating Accompanied by Chronic Constipation: A Prospective, Single-Center Randomized Open Trial," Current Therapeutic Research - Clinical and Experimental, vol. 77, pp. 58-62, 2015.

[12] T. Hasebe, N. Ueno, M. W. Musch et al., "Daikenchuto (TU100) shapes gut microbiota architecture and increases the production of ginsenoside metabolite compound K," Pharmacology Research \& Perspectives, vol. 4, no. 1, Article ID e00215, 2016.

[13] K. Togawa, J. Matsuzaki, M. Kobayakawa et al., "Association of baseline plasma des-acyl ghrelin level with the response to rikkunshito in patients with functional dyspepsia," Journal of Gastroenterology and Hepatology, vol. 31, no. 2, pp. 334-341, 2016.

[14] Y. Saegusa, T. Hattori, M. Nahata, C. Yamada, and H. Takeda, "A new strategy using rikkunshito to treat anorexia and gastrointestinal dysfunction," Evidence-Based Complementary and Alternative Medicine, vol. 2015, Article ID 364260, 10 pages, 2015.

[15] O. J. Kwon, M. Y. Kim, S. H. Shin et al., "Antioxidant and anti-inflammatory effects of rhei rhizoma and coptidis rhizoma mixture on reflux esophagitis in rats," Evidence-Based Complementary and Alternative Medicine, vol. 2016, Article ID 2052180, 13 pages, 2016. 


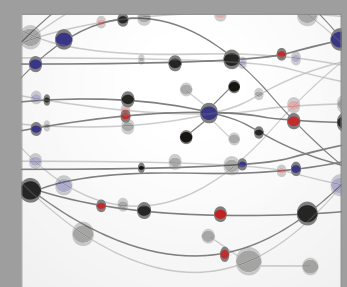

The Scientific World Journal
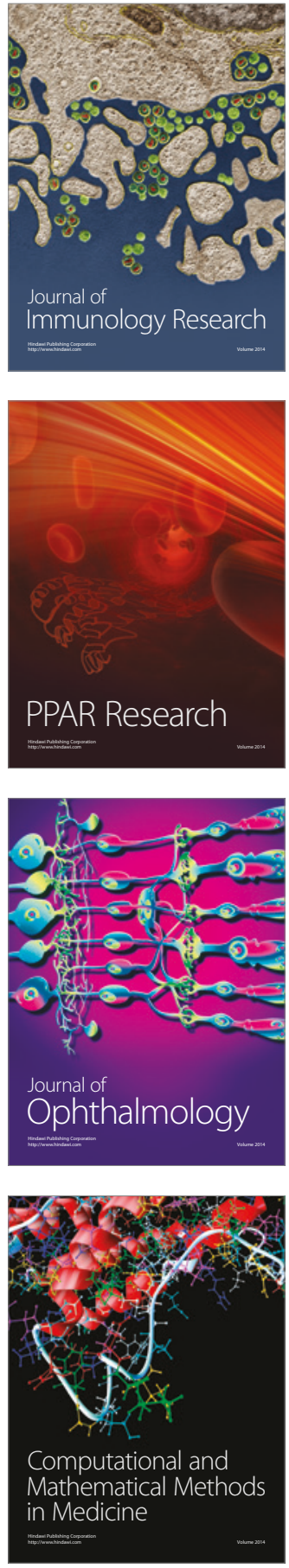

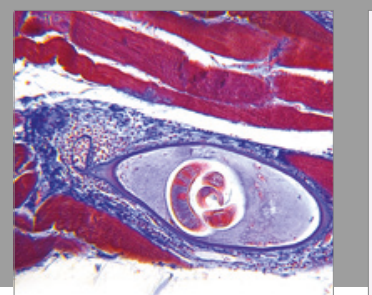

Gastroenterology Research and Practice
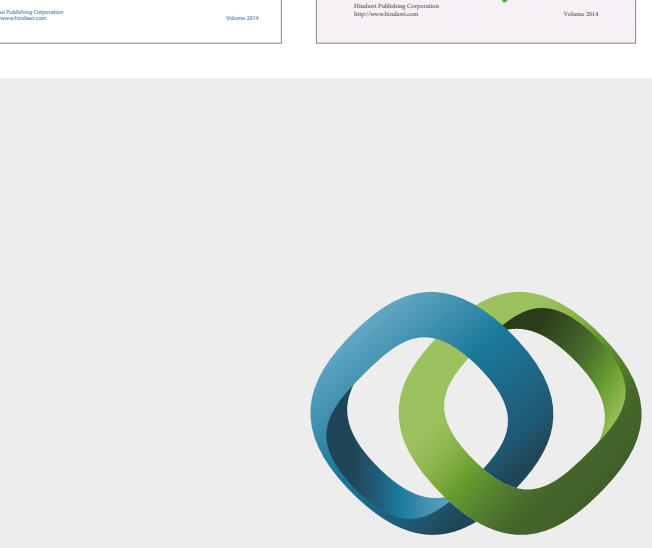

\section{Hindawi}

Submit your manuscripts at

https://www.hindawi.com
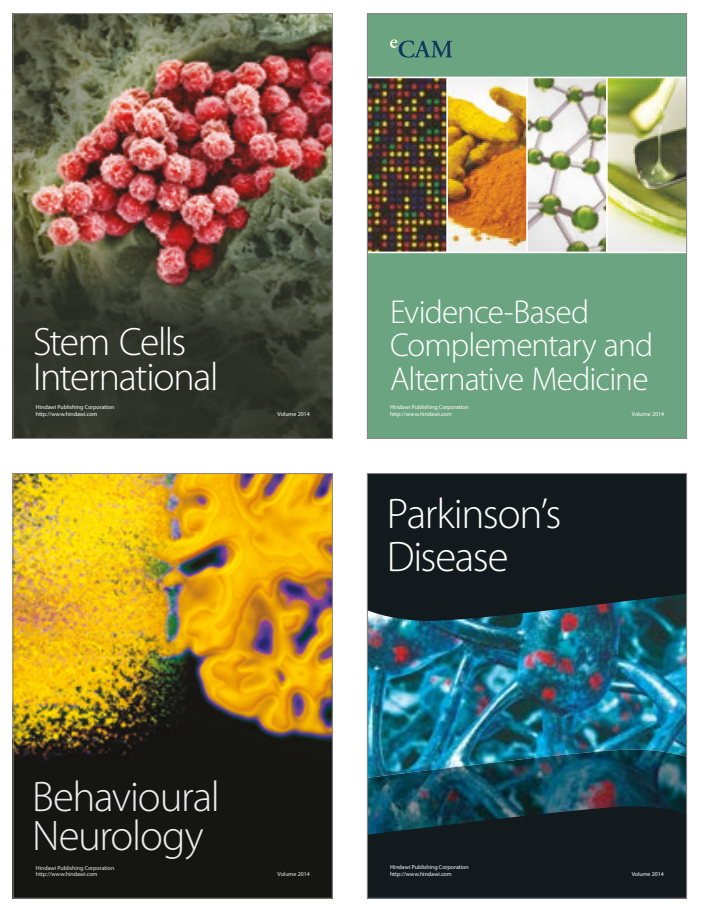
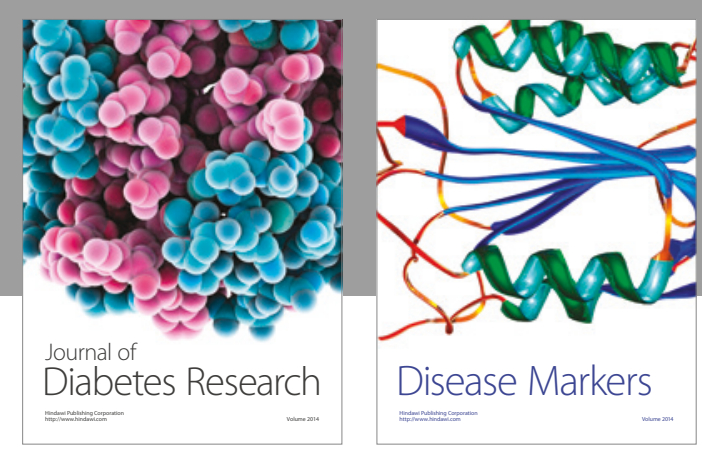

Disease Markers
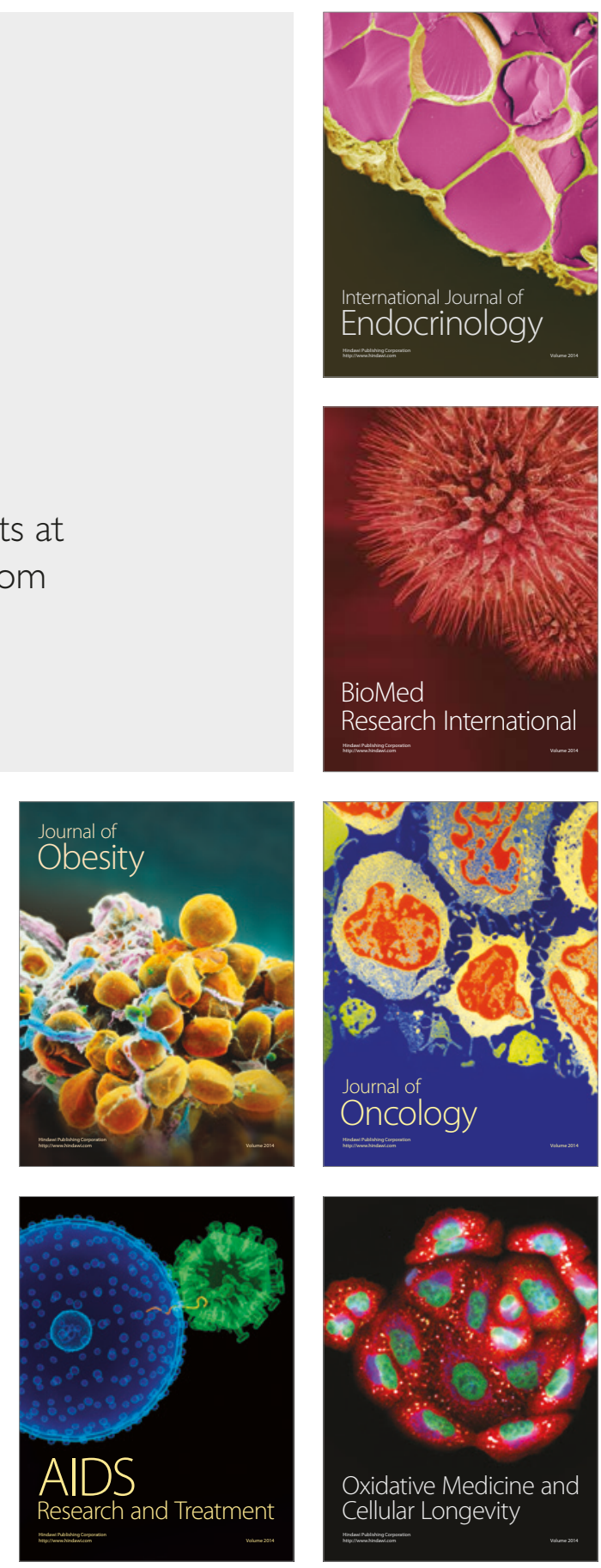